\title{
Using the method of postisometric relaxation for the technique of posers improvement of the Latin American program
}

\author{
Elena Propisnova ${ }^{1 *}$, Ekaterina Turchina ${ }^{1}$, Darya Degtyareva ${ }^{1}$, Elena Repnikova ${ }^{2}$ \\ ${ }^{1}$ Volgograd State Academy of Physical Culture, 400005, Volgograd, Russia \\ ${ }^{2}$ Vladimir State University named after Alexander and Nikolay Stoletov, 600000, Vladimir, Russia
}

\begin{abstract}
The aim of the research - to create and experimentally substantiate complexes of exercises, based on the method of postisometric relaxation, directed toward technical poses improvement of the Latin American program by highly-qualified dancers. Research methods: For the pedagogical experiment two groups of dancers were formed (control and experimental group) each group included 12 people. Unlike the control group the training process of dancers from the experimental group included the complexes of physical exercises based on the methodology of postisometric relaxation. Pedagogical experiment was held during 6 months (since September, 2018 till February, 2019). In terms of the research we measured the development level of flexibility of different muscles groups and defined the degree of mastering the technique of poses fulfillment by dancers. Research results. We created the complexes of exercises, based on the methodology of postisometric relaxation, directed toward technique of poses fulfillment of the Latin American program by athletes- dancers. According to the results of statistic data handling it was revealed that the created by us complexes had positive influence on spine and hip joints flexibility development. It provided poses fulfillment technique development during competitive activity of athletes-dancers from the experimental group. Conclusion. Thus, using the method of postisometric relaxation during the training process of athletesdancers provides purposeful increase of their technical mastery owing to qualitative poses fulfillment during competitive activity.
\end{abstract}

\section{Introduction}

Dancing sport is a competitive difficult coordinating activity with a subjective estimation of the results of 10 dances fulfillment by a dancing couple (European and the Latin American program), where judges have special criteria $[4,6,9,10]$. An optimal poses fulfillment in dancing compositions influences greatly the final result of competitive activity. This dependence is seen among highly-qualified athletes-dancers. They specialize in the Latin American program. From the point of view of technical fulfillment poses are estimated according to "technical quality" criterion and from the point of view of artistry- "choreography and presentation". Thus, technically correct poses fulfillment in the Latin American program dancing is reflected on the estimation of all criteria and influences the final result [1,6]. Having sufficient level of flexibility athletes-dancers can fulfill poses with maximal amplitude of movement. It is the base for correct technique, and it makes dancing sport spectacular. In dancing sets of a female partner plasticity is very important and is demonstrated more actively, but male partners also have many poses, where it is necessary to raise the leg higher than $90^{\circ}$, for example, fulfilling rondos in different positions of "rumba" or while fulfilling swing movements in "jive". Nowadays there are is a great diversity of methodologies directed toward the level of flexibility improvement. One of them is the method of postisometric relaxation. It provides muscles length and their elasticity increase and the exercises fulfillment happens without serious consequences [2,7,8]. It made us held a pedagogical experiment directed toward the technique of poses development in the Latin American program on the basis of postisometric relaxation method use.

\footnotetext{
* Corrsponding author: winston555@yandex.ru
} 


\section{Statement of problem}

The research works were held on the basis of dancing-sports club "Zhemchuzhina" in Volgograd, Russia. 22 highly-qualified athletes-dancers took part in the experiment (17-20 years-old). The athletes were divided into two subgroups - control and experimental group. Since September, 2018 till February, 2019 we used created complexes of exercises on the basis of postisometric relaxation method in the training process of highly-qualified athletes-dancers. Pedagogical testing was held in order to define the indices. They characterize flexibility development and in order to estimate the technique of poses fulfillment, included into competitive compositions of the Latin American program. Statistical material handling was realized using standard statistical programs [5].

\section{Results and discussion}

According to the literature sources and the experience of the leading specialists in the sphere of dancing sport we created poses classification, which had 4 groups: "Extensions", "Leans", "Swings", "Legs lifts". Also we defined the ways of their fulfillment (picture 1).

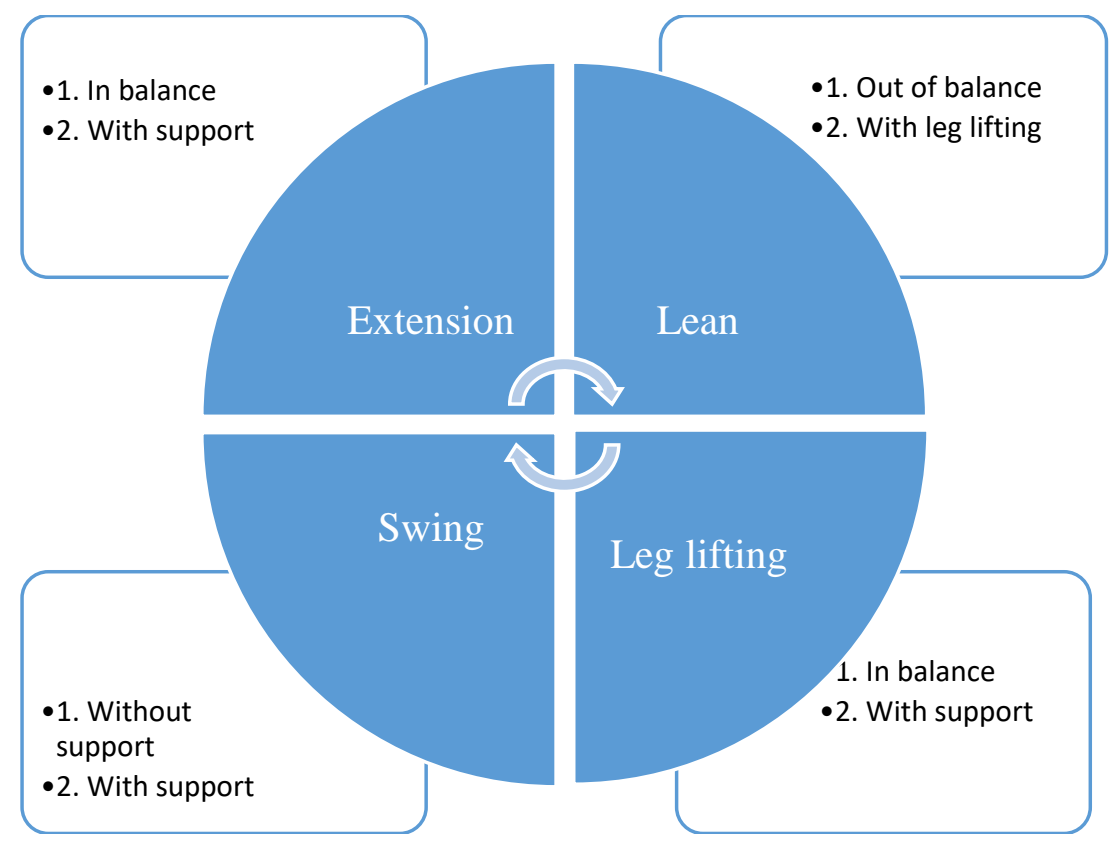

Fig. 1. Classification of the poses of the Latin American program

The poses of all groups can be fulfilled both "with support/without support" and "in balance/ out of balance". According to the direction poses in "Extension" are fulfilled only backwards, the exception is also "Swings" group, poses. They are not fulfilled in a circle other exercises are fulfilled in all directions. Then we defined combined poses, where the groups "Extensions" and Leans are combined with swings and legs lifting". This classification proves the fact that athletes-dancers should have an optimal level of flexibility.

Taking into account the results received during the preliminary pedagogical experiment and the results of scientific-methodical literature analysis we create the complexes of exercises based on postisometric relaxation method. These complexes were used during the final part of the training lesson in the experimental group.

The lessons were held three times a week, the duration of the final part was 30 minutes. The control group also had three lessons a week according to generally adopted program. During exercises fulfillment great attention was paid to breathing: during the breath isometric work was realized. It means muscles tension. On expiration the amplitude of movements was increased. As postisometric relaxation method is directed toward normal tonus of muscles renewal with the help of their relaxation. It is considered to be attenuated. Taking into account created by us classification and the defined on its basis poses, we selected exercises of postisometric character, directed toward flexibility improvement of the corresponding parts. They were responsible for qualitative body position realization [3, 8]. The created complexes included the exercises, which were divided into 2 groups. The first group included exercises directed toward flexibility of spine improvement. The second group includes exercises directed toward hip joint flexibility improvement.

Exercises for spine flexibility improvement:

\section{Body lifting}


I. p.- prone position, arms on the nape

- an athlete with the help of the supervisor fulfills body lifts till muscle resistance. A coach holds the dancer below on the shoulders;

- during inspiration an athlete fulfills a slight pressure into hands of a coach and stays for 6-10 seconds;

- during exhalation after pressure fulfillment, during relaxation, a coach strengthens the amplitude of the lean till an athlete feels not comfortable pain. Time of position holding -10 seconds.

\section{Body twisting}

I. p.- prone position, arms behind the head

- an athlete independently lifts the body from the floor $15^{\circ}$, a coach holds the hip with one hand, pressing down the floor. The second hand holds the athlete below on the shoulder twisting the body. Twisting happens till the athlete feels some resistance;

- an athlete fulfills some pressure on a coach's hand and stays for 6-10 seconds;

- after pressure fulfillment, during the moment of relaxation, a coach strengthens the amplitude of the body twist and sets the position during 10 seconds. The exercise is fulfilled on both sides.

\section{Body extension}

I. p.- knee stand

- an athlete stands on both knees facing the coach, who takes the same position and puts his hands from below on a dancer's shoulders. Back lean is fulfilled, the athlete's body uses the coach's body as a support. Extension happens till a coach feels some muscle resistance;

- an athlete fulfills some pressure upwards into a coach's hands and stays for 6-10 seconds;

- after the pressure fulfillment, during the moment of relaxation, the coach increases the amplitude of a back lean holding maximal position within 10 seconds.

\section{Leans}

I. p.- stand, hands on hips

- an athlete faces the coach, who sits on the chair and grips the dancer's hips and holds the position fixed. Then an athlete fulfills a lean. During the lean the opposite hand of the respondent raises over his head and follows body movement. One hand of a coach holds the hand on the hip down, the second hand fulfills some pressure on the other shoulder till feels some resistance;

- an athlete fulfills some pressure on the coach's lying hand near the shoulder and stays for 6-10 seconds;

- after pressure fulfillment a coach increases the amplitude of the lean and holds the position fixed during 10 seconds. The exercise is fulfilled on both sides.

\section{Twists}

I. p.- stand, arms held sideward

- an athlete faces the coach, who puts his right hand on the dancer's right shoulder and the left hand behind the right hip. Twist is fulfilled with the help of a coach's pressure on the shoulder, the left hand pulls the hip of an athlete on oneself. Twisting is realized till the coach feels some muscle resistance;

- an athlete fulfills some pressure on hands. He tries to return initial body position or when he can't fulfill simultaneous resistance, shoulder is fixed. Such position stays during 6-10 seconds;

- after pressure fulfillment, during the moment of relaxation, a coach increases the amplitude of twisting holding maximum set position within 10 seconds. The exercise is fulfilled on both sides.

\section{Exercises for hip joints flexibility and hip muscles plasticity development:}

\section{Side leg lift}

I. p. - lateral position

- an athlete in lateral position lifts a leg. He tries to put it behind the head. A coach presses down on the leg till he feels muscle resistance;

- then an athlete fulfills isometric work pressing with the leg down on a coach's hand. Such position is fixed for 610 seconds;

- after that there is passive relaxation, when muscles independently decrease the tonus, meanwhile a coach increases the amplitude owing to pressure increase. The exercise is fulfilled from two legs.

\section{Leg lift backwards}

I. p. - kneeling hips extended

- an athlete fulfills leg lift upwards. A coach stands behind and presses down on the leg till he feels muscle resistance;

- then an athlete fulfills an isometric work pressing down with the leg on a coach's hand. Such position is fixed for 6-10 seconds;

- after that there is passive relaxation, when muscles independently decrease the tonus. Meanwhile a coach increases the amplitude. The athlete should hold hip joint parallel to the floor. The exercise is fulfilled from two legs.

\section{Drawing leg aside}

I. p. - dorsal position

- an athlete draws his leg aside. The coach is before the athlete and pulls out the leg till he feels muscle resistance;

- then an athlete fulfills an isometric work pressing down with the leg on a coach's hand. Such position is fixed for 
6-10 seconds;

- after that there is passive relaxation, when muscles independently decrease the tonus, meanwhile a coach increases the amplitude fixing the second leg during 10 seconds. The exercise is fulfilled in turn for each leg.

\section{Hip joint stretching}

I. p. - kneeling hips extended

- an athlete puts one leg forward. The coach stands behind pressing down with his weight on the loins till he feels resistance;

- then an athlete fulfills an isometric work "pushing out" a coach's weight. Such position is fixed for 6-10 seconds;

- after that happens relaxation, muscle tonus decreases and the coach increases the amplitude, fixes the second leg and the lumbar cord. It is necessary not to turn the hip joint, to hold the pose during 10 seconds. The exercise is fulfilled in turn for each leg.

\section{Leg back abduction}

I. p. - prone position

- an athlete lifts the leg upward. The coach stands behind fixing the second leg on the floor. The coach lays a switch leg aside, forward holding on the hip and the knee. The pressure goes till muscle resistance and some pain in the inner part of the hip;

- then an athlete presses on a coach with the a switch leg. Such position is fixed for 6-10 seconds;

- after that passive relaxation happens and a coach increases the amplitude of movement using some pressure. The exercise is fulfilled in turn for each leg.

Each exercise was fulfilled 6-10 times. 60 training lessons were held during the experiment using the created complexes.

In order to estimate the effectiveness of the created complexes use we realized comparative analysis of indices. They characterized qualitative poses use of the Latin American program: the amplitude (flexibility level) and expert estimation of the fulfillment technique. Held at the beginning test didn't reveal valid differences in the studied indices between the control and the experimental groups $(p>0,05)$. It underlined homogeneity of the respondents [5]. After 6 months of constant training process we held the second test (table 1).

Table 1. Results of testing the development level of dancers' flexibility during the experiment

\begin{tabular}{|c|c|c|c|c|c|c|c|c|}
\hline \multirow[t]{2}{*}{ Tests } & \multicolumn{2}{|c|}{ Experimental group } & \multirow[t]{2}{*}{$\mathrm{t}$} & \multirow[t]{2}{*}{$\mathrm{p}$} & \multicolumn{2}{|c|}{ Control group } & \multirow[t]{2}{*}{$\mathrm{t}$} & \multirow[t]{2}{*}{$\mathrm{p}$} \\
\hline & $\begin{array}{l}\text { beforethe } \\
\text { experiment }\end{array}$ & $\begin{array}{c}\text { after the } \\
\text { experiment }\end{array}$ & & & $\begin{array}{l}\text { before the } \\
\text { experiment }\end{array}$ & $\begin{array}{l}\text { afterthe } \\
\text { experiment }\end{array}$ & & \\
\hline «Extension» (cm) & $64,25 \pm 9,2$ & $28,75 \pm 4,9$ & 3,41 & $<0,05$ & $62,16 \pm 6,13$ & $63,83 \pm 4,9$ & 0,95 & $>0,05$ \\
\hline 《Leans» $\left(^{0}\right)$ & $67,5 \pm 6,13$ & $84,16 \pm 4,6$ & 2,17 & $<0,05$ & $66,25 \pm 7,66$ & $72,08 \pm 4,6$ & 0,65 & $p 0,05$ \\
\hline 《Sideward swing » (0) & $87,5 \pm 6,13$ & $112,8 \pm 4,9$ & 3,18 & $<0,05$ & $78,33 \pm 6,13$ & $90,41 \pm 6,13$ & 1,09 & $>0,05$ \\
\hline 〈Split near the wall » $(\mathrm{cm})$ & $41,83 \pm 16,87$ & $22,5 \pm 3,37$ & 2,14 & $<0,05$ & $45,66 \pm 8,58$ & $36,16 \pm 3,37$ & 1,06 & $>0,05$ \\
\hline
\end{tabular}

Notes: $\mathrm{t}_{\text {table. }}=2,13$ in case $\alpha=0.05$.

Thus, the dynamics of flexibility level development indices in the experimental group has valid positive character, in comparison with the control group, changes in which are not proved by statistics.

The results of poses technique quality comparative analysis of the Latin American program (according to the results of expert estimation) in control and experimental groups are presented in table 2 . The level of technical readiness was estimated by three experts according to simple poses one element from each classification. The experts were highly-qualified judges in dancing sport. Control tests were estimated in points from «0» to «5» and were put individually according to each athlete.

Table 2. Comparative analysis of the Latin American program poses technique results in the experimental and control groups during the experiment

\begin{tabular}{|c|c|c|c|c|c|c|c|c|c|c|c|c|c|c|c|c|}
\hline \multirow[t]{3}{*}{ Tests } & \multicolumn{4}{|c|}{ «Extension» } & \multicolumn{4}{|c|}{ «Lean» } & \multicolumn{4}{|c|}{ «Swing» } & \multicolumn{4}{|c|}{ «Leg Lifting» } \\
\hline & \multicolumn{2}{|c|}{ EG } & \multicolumn{2}{|c|}{ KG } & \multicolumn{2}{|c|}{ EG } & \multicolumn{2}{|c|}{ KG } & \multicolumn{2}{|c|}{ EG } & \multicolumn{2}{|c|}{$\mathrm{KG}$} & \multicolumn{2}{|c|}{ EG } & \multicolumn{2}{|c|}{$\mathrm{KG}$} \\
\hline & 1 & 2 & 1 & 2 & 1 & 2 & 1 & 2 & 1 & 2 & 1 & 2 & 1 & 2 & 1 & 2 \\
\hline $\mathbf{X}$ & \multicolumn{2}{|c|}{5,53} & \multicolumn{2}{|c|}{4,31} & \multicolumn{2}{|c|}{5,86} & \multicolumn{2}{|c|}{3,87} & \multicolumn{2}{|c|}{5,84} & \multicolumn{2}{|c|}{4,01} & \multicolumn{2}{|c|}{5,39} & \multicolumn{2}{|c|}{4,17} \\
\hline $\mathbf{P}$ & \multicolumn{2}{|c|}{$<0,05$} & \multicolumn{2}{|c|}{$<0,05$} & \multicolumn{2}{|c|}{$<0,05$} & \multicolumn{2}{|c|}{$>0,05$} & \multicolumn{2}{|c|}{$<0,05$} & \multicolumn{2}{|c|}{$>0,05$} & \multicolumn{2}{|c|}{$<0,05$} & \multicolumn{2}{|c|}{$>0,05$} \\
\hline
\end{tabular}

Notes: 1 - before the experiment; 2 - after the experiment; $\mathrm{t}_{\text {table. }}=4,29$ in terms $\alpha=0.05$. 
Table shows that the results of all tests in the experimental group prove quality of poses improvement after the created by us complexes of exercises use. The changes are proved by statistics. The changes of "extension" test in the control group also were valid, however, according to other tests there were no valid changes. Thus, we can see the effectiveness of the created by us complexes of exercises, based on postisometric relaxation method, the use of which helped to increase the level of flexibility development among athletes-dancers. And as a result provided the technique of poses development in dancing of the Latin American program.

\section{Conclusions}

We created the classification of dancing poses. It is based on the content of the Latin American program dancing. We defined their summarizing characteristics. We revealed 4 kinds of main poses, which are the initial for many variations of their fulfillment. We introduced the complexes of exercises into educational-training process of highlyqualified athletes-dancers. These complexes are based on postisometric relaxation method and directed toward the technique of poses improvement of the Latin American program. The effectiveness of their use was proved and substantiated during the pedagogical experiment. The received results showed positive dynamics of the complexes influence on the level of flexibility development $(\mathrm{p}<0,05)$ and on the technique of poses development in dancing of the Latin American program $(\mathrm{p}<0,05)$.

\section{References}

1. N.N. Zakhareva, N.V. Kotenko, L.A. Belitskaya, E.R. Sokolova, Theory and practice of physical culture, 6, 45-49 (2012)

2. T. Myers. Anatomic trains (LLC “MERIDIAN-C”, Saint-Petersburg, 2012).

3. Aleksandr S. Kuznetsov, Zinaida M. Kuznetsova, Russian Journal of Physical Education and Sport, 14(4), 5-7 (2019)

4. E.V. Putintseva Works of All-Russian scientific-practical conference, dedicated to $50^{\text {th }}$ anniversary of Volgograd State Academy of Physical Culture (Volgograd State Academy of Physical Culture, Volgograd, 2010).

5. N.V. Stetsenko, I.A. Abdrakhmanova. Mathematical statistics (Volgograd State Academy of Physical Culture, Volgograd, 2019).

6. M.A. Terekhova Refereeing in dancing sport (Volgograd State Academy of Physical Culture, Volgograd, 2015).

7. E.V. Turchina, D.D. Kotova, D.I. Degtayrev, Theory and practice of physical culture, 11, 32 (2017).

8. E.V. Turchina, D.D. Kotova. The basis of myofascial relaxation (Volgograd State Academy of Physical Culture, Volgograd, 2019).

9. H Jaques The Theory and Practice of Revised Technique (MTA, London, 1998).

10. W. Laird Technique of Latin dancing (International dance publications, Brighton, 1990). 